\title{
Intramuscular viral delivery of paraplegin rescues peripheral axonopathy in a model of hereditary spastic paraplegia
}

\author{
Marinella Pirozzi, ${ }^{1}$ Angelo Quattrini,,2 Gennaro Andolfi,1 Giorgia Dina, ${ }^{2}$ \\ Maria Chiara Malaguti, ${ }^{2}$ Alberto Auricchio,, ${ }^{1}$ and Elena I. Rugarli1 \\ ${ }^{1}$ Telethon Institute of Genetics and Medicine (TIGEM), Naples, Italy. ${ }^{2}$ Department of Neurology, \\ University Vita-Salute, San Raffaele Scientific Institute, Milan, Italy.
}

\begin{abstract}
Degeneration of peripheral motor axons is a common feature of several debilitating diseases including complicated forms of hereditary spastic paraplegia. One such form is caused by loss of the mitochondrial energy-dependent protease paraplegin. Paraplegin-deficient mice display a progressive degeneration in several axonal tracts, characterized by the accumulation of morphological abnormal mitochondria. We show that adenoassociated virus-mediated (AAV-mediated) intramuscular delivery of paraplegin halted the progression of neuropathological changes and rescued mitochondrial morphology in the peripheral nerves of paraplegin-deficient mice. One single injection before onset of symptoms improved the motor performance of paraplegin-deficient mice for up to 10 months, indicating that the peripheral neuropathy contributes to the clinical phenotype. This study provides a proof of principle that gene transfer may be an effective therapeutic option for patients with paraplegin deficiency and demonstrates that AAV vectors can be successfully employed for retrograde delivery of an intracellular protein to spinal motor neurons, opening new perspectives for several hereditary axonal neuropathies of the peripheral nerves.
\end{abstract}

\section{Introduction}

Hereditary spastic paraplegia (HSP) is a neurodegenerative disorder characterized by progressive loss of motor axons of the corticospinal tracts and of sensory axons of the fasciculus gracilis by a dying-back mechanism (1-4). HSP patients complain of relentless weakness, spasticity, and impairment of the vibratory sense in the lower limbs and are ultimately confined to a wheelchair. Currently, this debilitating condition has no cure.

HSP affects 1 in every 10,000 individuals and is genetically heterogeneous, with 28 loci identified so far (5). Approximately $30 \%$ of HSP cases are inherited as autosomal-recessive traits. One of these recessive forms is due to loss-of-function mutations in the SPG7 gene, encoding paraplegin (6). Although the original family described with SPG7 mutations displayed a relatively pure phenotype, most cases are complicated by additional neurological symptoms such as cortical and cerebellar atrophy, optic atrophy, amyotrophia, and peripheral neuropathy (7).

Paraplegin is a subunit of an energy-dependent protease in the inner mitochondrial membrane (the m-AAA protease) (8). Studies in yeast have shown that the $\mathrm{m}$-AAA protease is involved in protein quality control activities in the inner mitochondrial membrane, such as ensuring the removal of nonassembled or misfolded membrane proteins and controlling ribosome assembly in mitochondria and thereby the formation of respiratory complexes and ATP synthase $(9,10)$.

We previously developed a mouse model for paraplegin deficiency by knocking out the first 2 exons of the mouse Spg7 gene (11). Paraplegin-deficient mice are characterized by a late-onset distal axo-

Nonstandard abbreviations used: AAV, adenoassociated virus; ALS, amyotrophic lateral sclerosis; GC, genome copies; HSP, hereditary spastic paraplegia.

Conflict of interest: The authors have declared that no conflict of interest exists.

Citation for this article: J. Clin. Invest. 116:202-208 (2006)

doi:10.1172/JCI26210. nopathy of spinal, optic, and peripheral axons, thus mimicking the human disease. Axonal degeneration is preceded by axonal swelling due to accumulation of organelles and neurofilaments, a sign of impaired axonal transport. Interestingly, paraplegin-deficient mice begin to show impaired motor performance on the rotarod apparatus at 4 months of age, 3 months before the first signs of pathology in the spinal cord are detected. The onset of the clinical phenotype correlates with the appearance of mitochondrial morphological abnormalities in synaptic terminals and in distal regions of spinal axons, suggesting that dysfunction of a subset of mitochondria is at the basis of the pathogenic process. This result is puzzling, since paraplegin is widely expressed in mitochondria from all tissues.

HSP due to paraplegin deficiency has several ideal features for exploiting a gene replacement therapeutic strategy: the onset is late, the progression of symptoms is relatively slow, and the molecular basis of the disease is caused by complete loss of function. The large window of time between the onset of motor symptoms and the actual loss of axons in the mouse model suggests that therapeutic options could be successfully exploited for several years after the disease is diagnosed. However, an open question is whether it is possible to successfully revert the accumulation of abnormal mitochondria in synaptic terminals of affected axons.

One challenge in designing therapies for neurological conditions based on gene delivery is the specific targeting of the affected neurons. Recently, a few studies have unraveled the ability of certain viral vectors to be retrogradely transported by axons, opening the path for novel routes of administration. Notably, both adenoassociated virus (AAV) vectors and lentiviral vectors based on equine infectious anemia virus have been shown to transduce spinal motor neurons after intramuscular injection (12-15).

In this study, we asked whether restoring paraplegin expression in mitochondria is efficient in preventing, halting, or reversing the mitochondrial abnormalities and the clinical and neuro- 


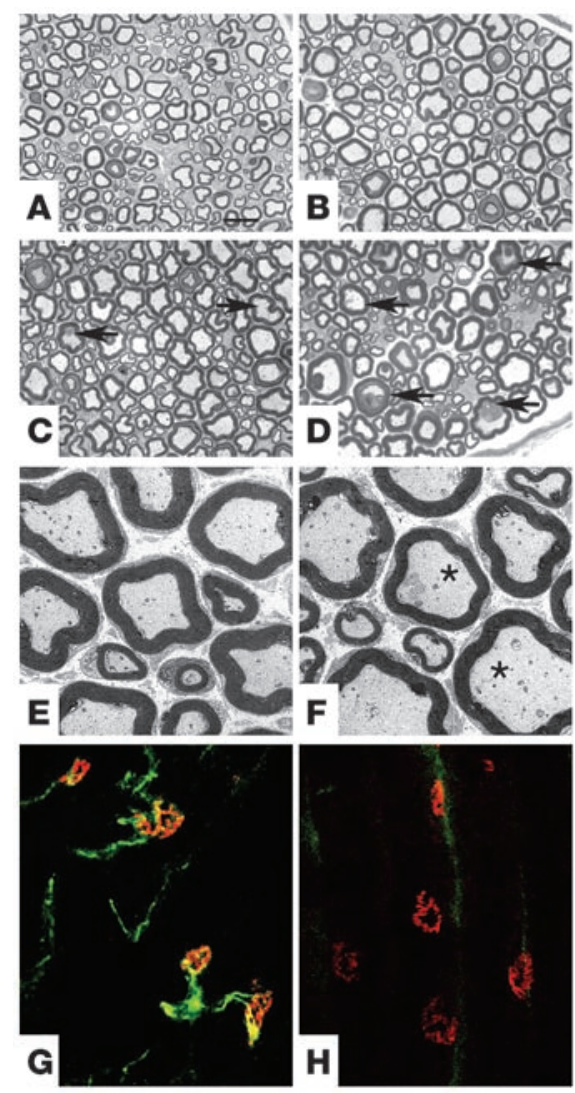

pathological phenotype of $S p g 7^{-/-}$mice. As a proof of principle, we resorted to $S p g 7$ gene replacement in the peripheral nerves by use of recombinant $\mathrm{AAV}$ vectors delivered intramuscularly. We found that this approach was successful in halting the progression of neuropathology in the peripheral nerves of paraplegin-deficient mice. Furthermore, mitochondrial morphological abnormalities were partially rescued. Surprisingly, $S p g 7$ gene replacement in the peripheral nerves before onset of symptoms ameliorated the rotarod performance of $S p \mathrm{p}^{-/-}$mice, suggesting that peripheral nerve involvement plays an important role in the manifestation of motor symptoms in the mouse model.

\section{Results}

Peripheral neuropathy in Spg7-/- mice. We previously described a progressive axonopathy in the sciatic nerve of $S p g 7^{-1-}$ mice (11). More in-depth analyses allowed us to detect a few axoplasmic alterations as early as 10 months of age in the distal nerve, while frank signs of degeneration appeared at 18 months (Figure 1, A-D). Axonal changes and degenerating axons were also observed at 18 months in the purely sensory saphenous nerve (data not shown), indicating that $S p g 7^{-1-}$ mice are affected by a motor-sensory peripheral neuropathy. We asked whether ultrastructural alterations of mitochondria precede these changes, as previously observed in the spinal cord (11). We found ultrastructural changes in sciatic nerve mitochondria of $S p g 7^{-1-}$ mice as early as 6 months of age, compared with age-matched controls (Figure 1, $\mathrm{E}$ and $\mathrm{F}$ ). The neuromuscular junctions appeared normal until 18 months of age, but were almost completely denervated at 24 months (Figure 1, G and $\mathrm{H}$ ).

\section{Figure 1}

Peripheral neuropathy in Spg $7^{-1-}$ mice. (A-D) Semithin sections of the distal sciatic nerve of a 6-month-old WT mouse (A) and Spg $7^{-/-}$mice at 6 months (B), 10 months (C), and 18 months (D) of age. Axonal changes, characterized by the accumulation of dense material (arrows), are appreciated in Spg $7^{-/-}$mice at 10 months and increase in severity at 18 months. (E and $\mathbf{F}$ ) Electron micrographs of the sciatic nerve of 6-month-old WT (E) and Spg $7^{--}$(F) mice. A few axons already contain abnormal mitochondria in Spg $7^{-1-}$ mice (asterisks). (G and $\mathbf{H}$ ) Confocal images of neuromuscular junctions from teased muscular fibers of 24-month-old WT (G) and Spg7-/- (H) mice double-labeled with rhodamine-tagged $\alpha$-bungarotoxin to stain acetylcholine receptors (red) and antibody to neurofilament NF200 to detect axons (green). Control preparations reveal the typical ( 1 axon per end plate) pattern of innervation, while in mutant mice the terminal plates are denervated. Scale bar: $15 \mu \mathrm{m}$ (A-D); $5 \mu \mathrm{m}$ (E and F). Magnification, $\times 63$ (G and $\mathbf{H})$.

Retrograde targeting of spinal motor neurons by intramuscular delivery of $A A V$ vectors. We tested the ability of AAV vectors derived from different serotypes (AAV2/1, AAV2/2, AAV2/5, AAV2/7, and AAV2/8, where the first number defines the genome and the second the capsid of origin) encoding for $\beta$-gal to efficiently transduce spinal motor neurons after a single unilateral intramuscular injection in the gastrocnemius muscles of adult mice. Four weeks after injection, the muscle and the spinal cord were analyzed for expression of the reporter gene. We found that both AAV2/1 and AAV2/2 transduced spinal motor neurons after intramuscular delivery (Figure 2). Transduction was restricted to the motor neurons corresponding to the injected side. Quantification revealed that up to 47 and 82 motor neurons in the lumbar spinal cord were positive for LacZ staining following intramuscular injection of LacZ-expressing vectors AAV2/1 and AAV2/2 (AAV2/1-LacZ and $\mathrm{AAV} 2 / 2-\mathrm{LacZ}$ ), respectively. These numbers are consistent with the labeling of a significant percentage of motor neurons innervating the gastrocnemius $(16,17)$. Notably, no known correlation exists between the efficiency of a given serotype of AAV to transduce the skeletal muscle and the retrograde transport to motor neurons.

To confirm that the transduction of spinal motor neurons derives from retrogradely transported viral vectors, we administered AAV2/1-LacZ intravenously. Although this procedure allowed efficient delivery of $\beta$-gal to the skeletal muscles (18), we did not observe transduction of spinal motor neurons (data not shown). These data confirm that the AAV vectors do not cross the adult blood-brain barrier and suggest that they must come in direct contact with the presynaptic terminals of projecting neurons to be internalized and retrogradely transported.

Intramuscular delivery of AAV2/2-Spg7 restores paraplegin expression in spinal cord mitochondria. Since paraplegin is totally deficient in Spg $7^{-1-}$ mice (11), we constructed AAV2/1 and AAV2/2 vectors expressing mouse Spg7 cDNA (AAV2/1-Spg7 and AAV2/2-Spg7) and tested their ability to reconstitute paraplegin expression in the skeletal muscle and the spinal cord of Spg $7^{-1-}$ mice following injection in the quadriceps and gastrocnemius muscles. Six months after injection, we isolated mitochondria from the skeletal muscle and spinal cord and assessed paraplegin expression by Western blot analysis. A protein corresponding to correctly processed paraplegin was present in skeletal muscle mitochondria in the case of both AAV2/1-Spg7 and AAV2/2-Spg7. In contrast, paraplegin was detected in spinal cord mitochondria only after AAV2/2 delivery, not AAV2/1 (Figure 3), probably due to the lower efficiency of the latter serotype in transducing the spinal motor neurons after intramuscular injection. 


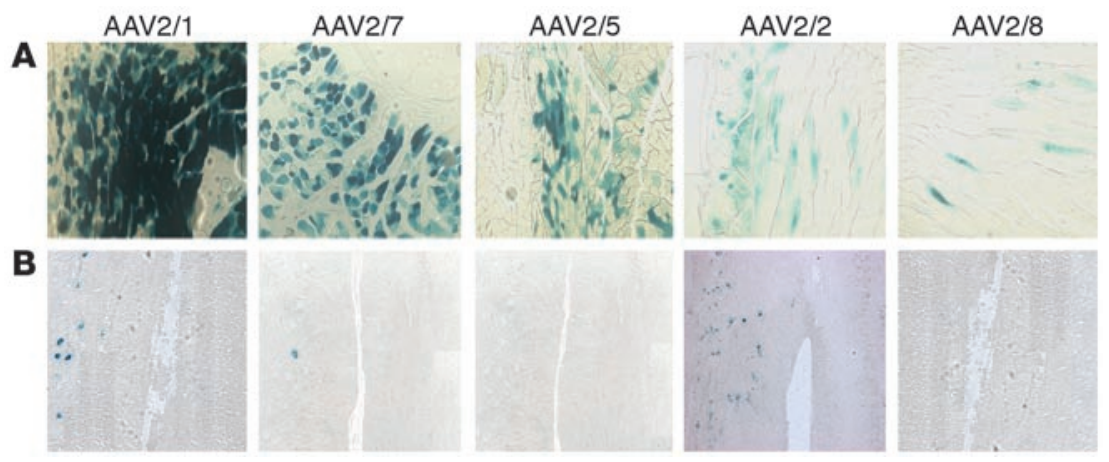

Figure 2

Retrograde transduction of spinal motor neurons by AAV vectors. Expression of $\beta$-gal activity in the skeletal muscle (A) and spinal cord (B) 4 weeks after injection of different AAV-LacZ vector serotypes in the gastrocnemius muscle. (A) AAV2/1 shows remarkable transduction of skeletal muscle, followed by AAV2/7 and AAV2/5. Conversely, the muscle transduction level for AAV2/2 and AAV2/8 is low. (B) Longitudinal sections of lumbar spinal cord to visualize targeting of motor neurons by retrograde transport of AAV (left side injected with AAV-LacZ). $\beta$-Gal activity restricted to motor neurons is visible only with AAV2/2 and AAV2/1. Magnification, $\times 10$.

$A A V$-Spg7 delivered to the skeletal muscle halts the progression of neuropathology in Spg7-1- mice. To explore the efficacy of Spg7 cDNA replacement in blocking the progression of the neuropathological cascade in $\operatorname{Spg} 7^{-1-}$ mice, we injected AAV2/2-Spg7 $(n=12)$ or AAV2/1-Spg7 $(n=8)$ unilaterally into both the quadriceps and the gastrocnemius of 10-month-old $S p g 7^{-/-}$mice. The contralateral side was injected with an AAV-LacZ vector to provide an internal control. We selected 10-month-old animals because at this age the motor phenotype was established and the first neuropathological changes in the peripheral nerves were limited but detectable (Figure 1C). In HSP patients this would correspond to an early symptomatic stage of the disease. The injected mice were kept for 6 months and then sacrificed for neuropathological analysis.

The effect of Spg7 replacement was first assessed in semithin sections of the distal part of the sciatic nerve. As expected, the sciatic nerve on the side injected with AAV-LacZ vector displayed several axons showing signs of axonopathy, such as the presence of inclusions and deposits, and a few degenerating axons (Figure 4, A and C). This phenotype was greatly reduced in the sciatic nerve corresponding to the side where the muscles had been injected with either AAV2/1-Spg7 or AAV2/2-Spg7 (Figure 4, B and D). To obtain a quantitative measure of the rescue of the phenotype, we performed a morphometric analysis and counted the percentage of axons showing unequivocal signs of axonopathy in random sections. Both AAV2/1-Spg7- and AAV2/2-Spg7-treated animals showed a statistically significant decrease in the number of affected axons in their sciatic nerves (Figure 4E). Consistent with the amelioration of the peripheral neuropathy, the skeletal muscle showed decreased signs of denervation in the treated side (Supplemental Figure 1; available online with this article; doi:10.1172/JCI26210DS1).

Paraplegin replacement rescues mitochondrial morphological abnormalities. In Spg $7^{-1-}$ mice, morphological abnormal mitochondria accumulated in axons of the sciatic nerve as early as 6 months of age (Figure $1 \mathrm{~F}$ ) and involved a consistent percentage of axons at 10 months, the age at which we injected the vectors. As the reason for the mitochondrial abnormalities is still poorly understood, an important question is whether they are reversible and can be rescued by restoring paraplegin function in mitochondria. To this end, we performed ultrastructural studies in the group of animals injected with AAV2/2 vectors and found that the number of axons containing abnormal mitochondria and accumulation of neurofilaments and other aggregates was significantly reduced in the sciatic nerve from the side treated with AAV2/2-Spg7 compared with the AAV-LacZ-injected side (Figure 5). Strikingly, quantification showed that the percentage of axons with abnormal mitochondria in the side treated with the AAV2/2-Spg7 vector was even significantly lower than that at the time the viral vector was administered, as assessed in untreated Spg $7^{-1-}$ mice at 10 months (Figure $5 \mathrm{H}$ ). This result strongly suggests that paraplegin delivery not only stops the progression of the mitochondrial phenotype, but also partially rescues the abnormal morphology of the organelles.

Replacement of paraplegin in the peripheral nerve is sufficient to improve motor performance. The neuropathological rescue following gene replacement in the peripheral nerve might influence the motor phenotype of $S p g 7^{-1-}$ mice. To address this issue, we turned to a different experimental paradigm, treating $S p g 7^{-1-}$ mice bilaterally at 3 months of age before any identifiable motor deficit and following their motor performance for several months through the rotarod test. The rotarod test is a useful means to assess the motor deficit of paraplegin-deficient mice from adulthood until about 15 months of age (11). After this age, the compliance of the mice lowers, regardless of genotype, and the test renders less reliable data.

We treated a cohort of $13 S p g 7^{-/-}$mice at 3 months of age bilaterally with AAV2/2-Spg7. As a control, another group of Spg $7^{-/-}$ mice $(n=13)$ were treated bilaterally with AAV-LacZ. At this age, the $S p g 7^{-1-}$ mice do not display any identifiable clinical or pathological phenotype. The motor performance of the 2 groups of mice was followed for 10 months through monthly rotarod tests and compared with that of $\operatorname{Spg} 7^{+/+}$animals of the same age $(n=9)$. Spg $7^{-1-}$ mice treated with the control AAV-LacZ vector started to show a decrease in their performance compared with WT animals
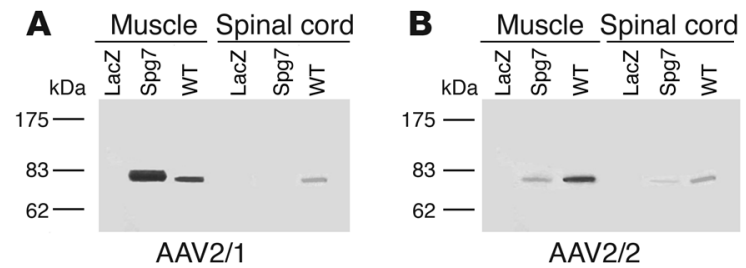

\section{Figure 3}

Paraplegin expression in skeletal muscle and spinal cord mitochondria after AAV-mediated intramuscular delivery of Spg7 cDNA. Western blot analysis of paraplegin on mitochondrial extracts from the gastrocnemius and the spinal cord of Spg $7^{-/-}$mice injected intramuscularly unilaterally with AAV2/1-Spg7 (A) and AAV2/2-Spg7 (B). In both cases, the contralateral side was injected with AAV-LacZ. For control we loaded mitochondrial extracts deriving from WT mice. With both serotypes, paraplegin is absent in muscles injected with AAV-LacZ but is restored upon injection of AAV-Spg7. The level of transduction in the muscle is higher when using AAV2/1, as expected. In the spinal cord, paraplegin is detected only when using AAV2/2-Spg7. 


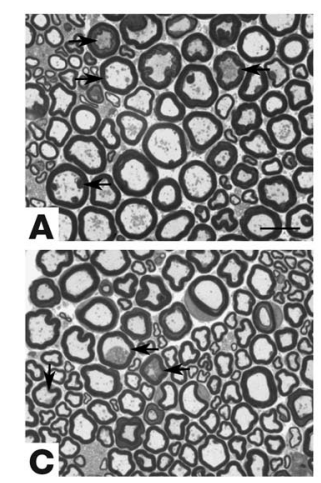

E

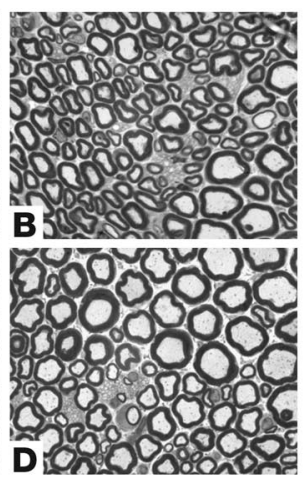

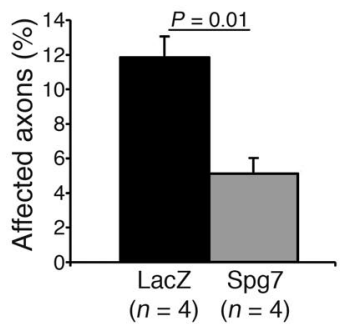

AAV $2 / 1$

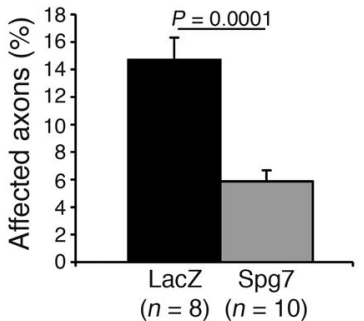

AAV $2 / 2$ at about 4 months (Figure 6A), as we previously described (11). Notably, we observed a statistically significant improvement of the rotarod performance in the group of $S p g 7^{-/-}$mice treated with the vector encoding paraplegin compared with those treated with the control AAV-LacZ vector (Figure 6A). This improvement was observed starting 2 months after treatment and was maintained for the entire period of observation.

To ascertain that the improvement in motor performance on the rotarod was due to a decrease in the number of affected axons in the treated animals, we analyzed their sciatic nerves and performed a morphometric analysis. We found that the percentage of axons showing neuropathological changes in the sciatic nerves of animals injected with AAV-Spg7 was significantly less than that observed in the mice treated with AAV-LacZ (Figure 6B).

Since the sciatic nerve is a mixed motor and sensory nerve, we wanted to determine whether part of the therapeutic effect is due to the rescue of sensory neurons. Paraplegin-deficient mice are

\section{Figure 5}

Rescue of the ultrastructural mitochondrial abnormalities. (A-G) Electron micrographs of the sciatic nerves in 16-month-old Spg $7^{-1-}$ mice. Mice were analyzed 6 months after intramuscular injection with AAVLacZ on the left side (A, C, and F) and AAV2/2-Spg7 on the right side (B, D, E, and G). A significant reduction in the number of axons containing abnormal mitochondria is observed in the sciatic nerve from the side injected with the AAV2/2-Spg7 vector (B). Giant mitochondria with disrupted cristae and glycogen accumulation (asterisk in F) are detected in the axons from the side treated with the control vector ( $\mathbf{C}$ and $\mathbf{F}$ ), while normal mitochondria with well-preserved cristae are present on the contralateral side (D, E, and $\mathbf{G})$. (H) Morphometric quantification of the percentage of sciatic nerve axons with abnormal mitochondria. Upon injection of AAV2/2-Spg7, the number of axons with abnormal mitochondria significantly decreases compared with the contralateral side and drops below the levels that were present at the time of injection. The $P$ values of Student's $t$ tests are shown. Scale bar: $5 \mu \mathrm{m}$ (A and B); $1.5 \mu \mathrm{m}$ (C, D, and F); $1 \mu \mathrm{m}$ (E); $400 \mathrm{~nm}$ (G).

\section{Figure 4}

Rescue of the neuropathological phenotype in the peripheral nerve. (A-D) Semithin sections of the distal sciatic nerves in 16-month-old Spg $7^{-1-}$ mice. Six months before the neuropathological studies, mice were treated with a single intramuscular injection of AAV-LacZ on the left side (A and $\mathbf{C})$ and either AAV2/1-Spg7 (B) or AAV2/2-Spg7 (D) on the right side. Note the decreased number of affected axons (arrows) in the nerves from the side treated with the Spg7 vectors. (E) Morphometric quantification of the percentage of affected axons for animals treated with AAV2/1 and AAV2/2 vectors. The $P$ value of Student's $t$ test is shown. Scale bar: $15 \mu \mathrm{m}(\mathbf{A}-\mathbf{D})$.

indeed affected by a progressive retrograde axonopathy of sensory fibers in peripheral nerves and in spinal central pathways (ref. 11 and data not shown). A subset of these fibers, arising in the muscle spindles and conveying the sense of joint position, may have been targeted after intramuscular delivery of the viral vector. Some of these afferent fibers enter the spinal cord from the dorsal roots and ascend the spinal cord directly in the fasciculus gracilis. To this end, we performed semithin sections and ultrastructural studies of the fasciculus gracilis at cervical level in the same 2 groups of animals that were subjected to the rotarod analysis. These animals were injected bilaterally at 3 months and analyzed at 13 months. We did not find any statistically significant improvement in the cohort of mice treated with AAV-Spg7 versus AAV-LacZ (data not shown).
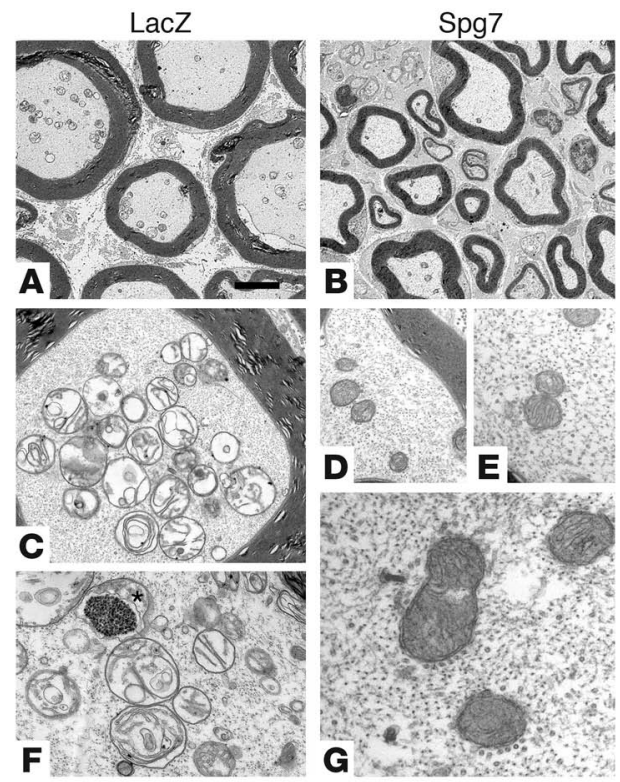

H

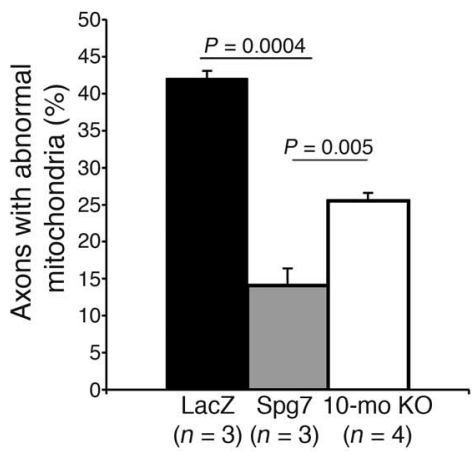




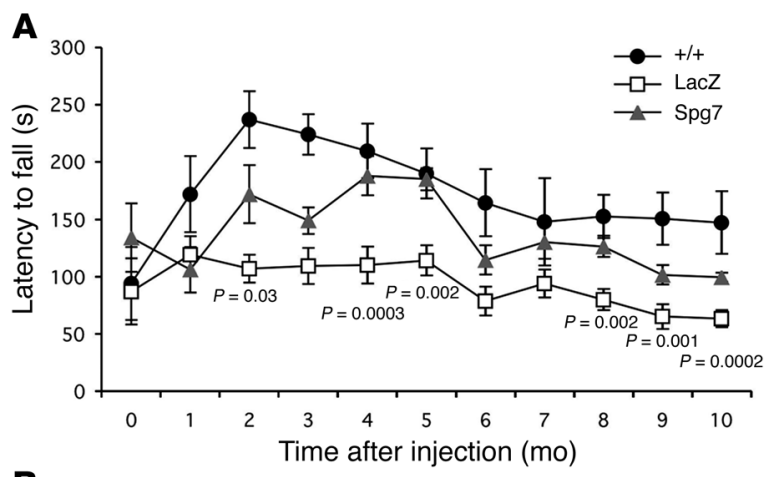

B

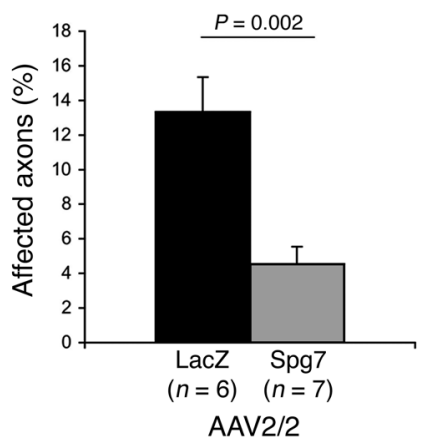

Taken together, our data allow us to conclude that retrograde delivery of paraplegin to spinal motor neurons through AAV vectors has an impact on the motor phenotype in paraplegin-deficient mice.

\section{Discussion}

Gene transfer to the peripheral nervous system has great therapeutic potential for the treatment of many neurological diseases, such as spinal muscular atrophy, amyotrophic lateral sclerosis (ALS), hereditary axonal motor neuropathies, and HSP. These diseases are characterized by degeneration of cell bodies and axons of spinal motor neurons of varying severity. Beside palliative treatments, no efficacious therapy is available yet. We used a HSP mouse model, due to the lack of the mitochondrial protein paraplegin, to exploit the efficacy of AAV-mediated gene delivery in the skeletal muscle to rescue degeneration of motor peripheral axons. Our data show that intramuscular administration of AAV-Spg7 in Spg $7^{-/-}$mice resulted in reexpression of paraplegin in spinal cord mitochondria, rescued the neuropathological changes, and improved the motor performance on the rotarod.

$A A V$ vectors have several desirable features for the transduction of neurons of the central and peripheral nervous system, such as derivation from a nonpathogenic virus and capability to infect postmitotic cells and to mediate sustained expression after single administration $(19,20)$. For these reasons, AAV vectors are being tested in human clinical trials (21). Previous studies have reached somewhat conflicting results on the efficacy of retrograde transduction of spinal motor neurons following the injection of AAV vectors in the skeletal muscle $(13,22)$. The most successful study has used AAV2/2 vectors to deliver IGF-1 in a mouse model of ALS, showing a significant prolongation of survival after treatment (13). Since IGF-1 is a secreted neurotrophic factor, the possibility remains that part of the therapeutic effect might be due to secretion of IGF-1 either from the muscle or from a low number of transduced motor neurons. Indeed, an increase in survival similar to that observed after AAV intramus-

\section{Figure 6}

Intramuscular viral delivery of paraplegin improves the motor performance of Spg $7^{-/-}$mice. (A) Monthly performance of Spg $7^{+/+}$mice, AAV-LacZ-treated Spg $7^{-/-}$mice, and AAV2/2-Spg7-treated Spg $7^{-/-}$ mice on an accelerating rotarod apparatus beginning at 3 months, the time of the bilateral viral injection. The mice treated bilaterally with the AAV-Spg7 vector showed a progressive better performance compared with Spg $7^{-/-}$mice injected bilaterally with the AAV-LacZ vector. Where significant, $P$ values for individual Student's $t$ test between the 2 groups of treated $S p g 7^{-/-}$mice are shown. In addition, mice treated with AAV2/2-Spg7 displayed a statistically significant difference in their performance during the course of the experiment (1-way ANOVA, $P=0.0001$ ), as did the control mice (1-way ANOVA, $P=0.008$ ), in contrast to mice injected with AAV-LacZ (1-way ANOVA, $P=0.07$ ). (B) Morphometric quantification of the percentage of affected axons in the sciatic nerves 10 months after treatment with AAV-LacZ or AAV2/2-Spg7 shows a statistically significant decrease due to the treatment. The $P$ value of Student's $t$ test is shown.

cular administration was noted in ALS mice crossed with transgenic mice expressing an IGF-1 isoform under a muscle-specific promoter, suggesting that release of IGF-1 from the muscle is sufficient for achieving some therapeutic effect in ALS mice (23).

We tested the ability of different AAV serotypes to retrogradely transduce spinal motor neurons. In agreement with previous observations (13), our data show that both AAV2/2 and AAV2/1 were retrogradely transported to spinal motor neurons after intramuscular injection. Several lines of evidence support our claim of retrograde targeting of spinal motor neurons. First, the AAV-LacZ vectors were always injected in the skeletal muscle unilaterally, and $\beta$-gal staining was restricted to the motor neurons of the injected side. Second, we found that transduction of motor neurons was not achieved after intravenous delivery of high doses of the AAV2/1 vector, indicating that it does not cross the adult blood-brain barrier. Finally, paraplegin is an intracellular protein, a subunit of the mitochondrial $\mathrm{m}$-AAA protease in the inner mitochondrial membrane, which excludes the possibility that part of the therapeutic effect may be due to long-range secretion. Therefore the successful rescue of the sciatic nerve axonopathy, which occurred only in the side injected with AAV-Spg7, is by itself strong evidence for retrograde transport of the viral vector. Although AAV2/2 was slightly more efficient for retrograde transduction of motor neurons in our $\beta$-gal targeting experiments and Western blot analysis, we observed similar therapeutic effects in $S p g 7^{-1-}$ mice with both viral serotypes, suggesting that restoration of even a very low amount of paraplegin might be sufficient. This is in line with the fact that paraplegin is involved in a recessive form of HSP, due to complete loss of function of the protein. In conclusion, this study provides the first evidence to our knowledge that an intracellular protein can be targeted to motor neurons after intramuscular injection of AAV vectors, indicating that they should definitively be considered an alternative possibility to equine infections anemia viral vectors for this application.

The results of our study also provide novel indications as to the biology underlying HSP and its clinical aspects. We injected AAVSpg7 in the skeletal muscles of knockout mice at 10 months of age. Although only a few signs of axonopathy are evident in the peripheral nerve by light microscopy at this age, abnormal mitochondria had been accumulating in axons for several months (Figure 1, B and F). This age was chosen because it likely corresponds to an early symptomatic stage of the disease in human patients, a time when starting a therapy would be appropriate. We found that we successfully 
stopped the progression of the neuropathological changes in the peripheral nerves. This result is especially striking at the ultrastructural level. In fact, the number of axons showing abnormal mitochondria was significantly reduced in the sciatic nerve of the treated side compared with the control side and dropped even below the levels present at the time when the vector was administered.

Based on these results, we conclude that restoration of low levels of paraplegin is sufficient to rescue the mitochondrial morphology defect, indicating that this phenotype is reversible in principle. Indeed, mitochondria are highly dynamic organelles, whose shape and function are subject to regulation. Notably, defects in genes regulating mitochondrial morphology are emerging as an important cause of neuropathy in humans (24-26), suggesting that complex control of mitochondrial morphology may exist in neurons and may be important for axonal function. Furthermore, we noticed that the accumulation of mitochondria previously observed in affected axons (11) was reduced in the sciatic nerves from the AAV-Spg7treated side, suggesting that rescue of the mitochondrial morphology corresponds with an improvement of axonal transport.

Delivery of paraplegin in Spg7 $7^{-1-}$ mice at 3 months of age ameliorated their motor performance on the rotarod. This result was unexpected, since our approach transduced only spinal motor neurons but failed to target pyramidal and extrapyramidal motor neurons and sensory neurons, whose axons continued to degenerate in these animals (E.I. Rugarli and A. Quattrini, unpublished observation). We think it is unlikely that the expression of high levels of paraplegin in the muscle is sufficient to improve motor ability. In fact, although paraplegin is ubiquitously expressed, its loss causes a cell-autonomous, neuronal-specific phenotype. Paraplegin-deficient mice do not show any myopathic phenotype apart from signs of denervation (11). These data therefore provide a strong indication that involvement of peripheral motor axons is an important clinical component that should be investigated more in depth in human patients. Indeed, in several other complicated forms of HSP, amyotrophia and peripheral motor neuropathy are prominent features, suggesting that involvement of spinal motor neurons is not uncommon in HSP (27-29). The lag of time that occurs between viral injection and motor improvement is attributable to the time for both retrograde uptake of the virus and paraplegin expression and transport of mitochondria along the axons.

In conclusion, our study provides a proof of principle that restoration of a low amount of paraplegin in affected neurons is extremely efficacious in blocking the progression of the disease, even after onset of the pathological process. Due to the mild phenotype of our HSP model, we were able to analyze the animals for phenotype rescue several months after a single administration of the therapeutic vector, showing unequivocally that $\mathrm{AAV}$ vectors sustain prolonged infection in neuronal cells. This approach can potentially be transferred with success to other motor axonal neuropathies of genetic origin.

\section{Methods}

Recombinant AAV vector construction and production. The mouse Spg7 coding sequence was cloned in the NotI-BamHI sites of the pAAV2.1-CMV-EGFP plasmid to obtain the pAAV2.1-CMV-Spg7 plasmid, which was triple transfected in 293 cells for recombinant AAV2/1- and AAV2/2-CMV-paraplegin production as previously described (30). The AAV2/1-, AAV2/2-, AAV2/5-, AAV2/7-, and AAV2/8-CMV-LacZ vectors were also produced by triple transfection as previously described (30-32). AAV2/1, AAV2/5, $\mathrm{AAV} 2 / 7$, and AAV2/8 vectors were purified by $\mathrm{CsCl}_{2}$ centrifugation, while AAV2 vectors were produced by heparin affinity purification. Physical titers of AAV vector preparations were assessed by real-time PCR (33) and were expressed as genome copies (GC) per milliliter. The vectors used in this study were produced by the TIGEM-AAV Vector Core.

Intramuscular and intravascular delivery of AAV-LacZ vectors. All animal procedures were approved by the Institutional Animal Care and Use Committee of Cardarelli Hospital (Naples, Italy). One-month-old C57BL/6 mice (at least 3 animals for each serotype) were anesthetized with avertin, and an incision 2-3 mm long was made in the skin to expose the gastrocnemius muscle. A total dose of $10^{11} \mathrm{GC}$ of each vector preparation was injected into 3 different sites of the gastrocnemius ( 3 injections of $30 \mu \mathrm{l} \mathrm{each}$ ) using a $100-\mu 1$ Hamilton syringe. Alternatively, $10^{12}$ GC of AAV2/ 1 was injected in the tail veins of C57BL/ 6 mice $(n=3)$.

For LacZ staining, animals were sacrificed 4 weeks after injection and perfused transcardially with $2 \%$ PFA/ $0.5 \%$ glutaraldehyde in PBS. The tissues were cryoprotected in $30 \%$ sucrose solution and processed for X-gal staining using standard techniques, either as whole-mount preparations (skeletal muscle) or after cryosectioning (spinal cord).

In vivo treatment with $A A V-S p g 7$ vectors. Mice were anesthetized with avertin, and an incision 2-3 $\mathrm{mm}$ long was made in the skin to expose the gastrocnemius and quadriceps muscles. A total dose of $10^{11} \mathrm{GC}$ of each vector preparation was injected into 3 different sites of the gastrocnemius and quadriceps ( 3 injections of $30 \mu \mathrm{l}$ each) using a 100- $\mu$ l Hamilton syringe.

Mice were treated following 2 experimental protocols. In the first experimental setting, 10-month-old Spg7-/- mice were injected unilaterally either with AAV2/2-Spg7 $(n=12)$ or AAV2/1-Spg7 $(n=8)$. In both cases, the contralateral side was injected with AAV-LacZ vector for an internal control. Animals were kept for 6 months before being sacrificed for Western blot, histological, and ultrastructural analysis. Age at the time of analysis was 16 months.

In the second experimental setting, 3-month-old Spg $7^{-/-}$mice $(n=13)$ from different litters were treated bilaterally with AAV2/2-Spg7. A control group of $S p g 7^{-1}$ mice of the same age $(n=13)$ was treated bilaterally with AAV-LacZ. All mice were tested in the rotarod apparatus once a month for the next 10 months, and their performance was compared with that of an age-matched group of $S p g 7^{+/+}$mice $(n=9)$. After 10 months, AAV-Spg7and AAV-LacZ-treated mice were sacrificed for histological analysis to quantify the rescue of the neuropathological phenotype. Age at the time of histological analysis was 13 months.

Western blot. Western blot analysis was performed on Spg $7^{-/-}$mice injected unilaterally in the gastrocnemius and the quadriceps muscles with AAV2/1-Spg7 $(n=2)$ and AAV2/2-Spg7 $(n=1)$ at 10 months and sacrificed 6 months afterward (see above). Mitochondria were isolated from the gastrocnemius muscle and the lumbar spinal cord as previously described (11). Approximately $50 \mu \mathrm{g}$ of mitochondrial proteins was separated by SDS-PAGE and blotted onto nitrocellulose. Filters were incubated with a specific antiparaplegin antiserum (11).

Neuromuscular staining. Isolated muscles were fixed in $4 \%$ paraformaldehyde in $0.1 \mathrm{M}$ PBS for 15 minutes. The muscles were then teased apart and incubated in $0.1 \mathrm{M}$ PBS for 10 minutes to remove the fixative before labeling acetylcholine receptors by incubating on a shaker for 20 minutes at room temperature in the presence of $1 \mathrm{mg} / \mathrm{ml} \alpha$-bungarotoxin conjugated to tetramethyl-rhodamine isothiocyanate (Invitrogen Corp.). After 3 washes with PBS, muscles were first permeabilized with $0.5 \%$ Triton X-100 (Sigma-Aldrich) in blocking buffer (10\% goat serum in PBS) for 30 minutes at room temperature and then incubated in primary monoclonal antibody direct against $200-\mathrm{kDa}$ neurofilament protein (NF200, phosphorylated and nonphosphorylated; Sigma-Aldrich) diluted 1:400 in $1 \%$ goat serum in PBS on a shaker at $4^{\circ} \mathrm{C}$ overnight. The following morning, the preparations were washed in PBS and incubated on a shaker for 4 hours at room temperature in $1 \%$ goat serum in PBS containing 1:100 dilution of goat anti-mouse antibody conjugated to the fluorescence-labeled FITC (Dako). Preparations were once more washed 3 
times in PBS, mounted in Vectashield on standard glass slides (Vector Laboratories), and examined under a confocal scanning laser microscope.

Neuropathology. Semithin and ultrathin morphological analyses were conducted as described previously $(34,35)$. Mice were anesthetized intraperitoneally with avertin and perfused with $4 \%$ paraformaldehyde in PBS. The sciatic nerves and the spinal cord were removed, postfixed in $0.12 \mathrm{M}$ phosphate buffer and $2 \%$ glutaraldehyde, and then sectioned transversely into 2-mm blocks, postfixed with osmium tetroxide, and embedded in Epon (Sigma-Aldrich). Cross sections were cut from the distal part of the sciatic nerve and the cervical part of the spinal cord.

Morphometric analyses. The percentage of abnormal axons in semithin sections was counted in 3 random sections per sciatic nerve. At least 300 axons were scored for each nerve. For quantification of ultrastructural mitochondria abnormalities, an average of 10 fields per nerve was analyzed. In each case, we scored at least 100 axons. Analyses were performed on photographs captured using an electron microscope (magnification, $\times 3,000$ ). All morphometric counts were performed in a blinded and nonbiased manner, with the treatment of animal unknown to the experimenter.

Rotarod. Motor performance was evaluated with a Rota-Rod apparatus (accelerating model; Ugo Basile). Each Spg $7^{-/}$experimental group consisted of 13 animals derived from different litters. The group of WT mice consisted of 9 mice. All the animals were 3 months old when the analysis started. All animals were tested on the rotarod once a month from the time of injection. Mice were placed on the accelerating rod at a starting speed of $4 \mathrm{rpm}$, reaching a final speed of $40 \mathrm{rpm}$ in 5 minutes. The animals were allowed to stay on the rod for a maximum of 300 seconds, and the time of hold on the rod was scored.
Statistics. Data are expressed as the mean \pm SEM. Statistical analysis of morphometric measurements was performed using a 2-tailed unpaired Student's $t$ test. Statistical analysis of rotarod activity was performed by 2-tailed unpaired Student's $t$ test at each time point between the AAV-LacZand AAV-Spg7-treated groups. In addition, we performed 1-way ANOVA for comparison of the rotarod performance over the course of study within each experimental group.

\section{Acknowledgments}

The authors wish to thank Diego Di Bernardo, Graciana DiezRoux, and Enrico Surace for helpful comments and discussion. This work has been supported by Telethon Foundation and Muscular Dystrophy Association grants (to E.I. Rugarli) and by Diagnostic Molecular Imaging (DiMi) Network of Excellence of the European Community (to A. Auricchio).

Received for publication July 8, 2005, and accepted in revised form October 18, 2005.

Address correspondence to: Elena I. Rugarli, Division of Biochemistry and Genetics, National Neurological Institute, via Temolo 4, 20126, Milan, Italy. Phone: 39-02-23942614; Fax: 39-02-23942619; E-mail: rugarli@istituto-besta.it.

Elena I. Rugarli's present address is: Division of Biochemistry and Genetics, National Neurological Institute, Milan, Italy.
1. Harding, A.E. 1983. Classification of the hereditary ataxias and paraplegias. Lancet. 1:1151-1154.

2. Harding, A.E. 1984. Hereditary "pure" spastic paraplegia. In The hereditary ataxias and related disorders. Churchill Livingstone. New York, New York, USA. 174-191.

3. Reid, E. 2003. Science in motion: common molecular pathological themes emerge in the hereditary spastic paraplegias. J. Med. Genet. 40:81-86.

4. Schwarz, G.A., and Liu, C.N. 1956. Hereditary (familial) spastic paraplegia: further clinical and pathological observations. Arch. Neurol. Psychiatry. 75:144-162.

5. Fink, J.K. 2003. Advances in the hereditary spastic paraplegias. Exp. Neurol. 184(Suppl. 1):S106-S110.

6. Casari, G., et al. 1998. Spastic paraplegia and OXPHOS impairment caused by mutations in paraplegin, a nuclear-encoded mitochondrial metalloprotease. Cell. 93:973-983.

7. Wilkinson, P.A., et al. 2004. A clinical, genetic and biochemical study of SPG7 mutations in hereditary spastic paraplegia. Brain. 127:973-980.

8. Langer, T. 2000. AAA proteases: cellular machines for degrading membrane proteins. Trends Biochem. Sci. 25:247-251.

9. Langer, T., Kaser, M., Klanner, C., and Leonhard, K. 2001. AAA proteases of mitochondria: quality control of membrane proteins and regulatory functions during mitochondrial biogenesis. Biochem. Soc. Trans. 29:431-436.

10. Nolden, M., et al. 2005. The m-AAA protease defective in hereditary spastic paraplegia controls ribosome assembly in mitochondria. Cell. 123:277-289.

11. Ferreirinha, F., et al. 2004. Axonal degeneration in paraplegin-deficient mice is associated with abnormal mitochondria and impairment of axonal transport. J. Clin. Invest. 113:231-242. doi:10.1172/ JCI200420138.

12. Mazarakis, N.D., et al. 2001. Rabies virus glycoprotein pseudotyping of lentiviral vectors enables retrograde axonal transport and access to the nervous system after peripheral delivery. Hum. Mol. Genet.
10:2109-2121.

13. Kaspar, B.K., Llado, J., Sherkat, N., Rothstein, J.D., and Gage, F.H. 2003. Retrograde viral delivery of IGF-1 prolongs survival in a mouse ALS model. Science. 301:839-842.

14. Azzouz, M., et al. 2004. Lentivector-mediated SMN replacement in a mouse model of spinal muscular atrophy. J. Clin. Invest. 114:1726-1731. doi:10.1172/ JCI200422922.

15. Azzouz, M., et al. 2004. VEGF delivery with retrogradely transported lentivector prolongs survival in a mouse ALS model. Nature. 429:413-417.

16. LaMonte, B.H., et al. 2002. Disruption of dynein/ dynactin inhibits axonal transport in motor neurons causing late-onset progressive degeneration. Neuron. 34:715-727.

17. Mohajeri, M.H., Figlewicz, D.A., and Bohn, M.C. 1998. Selective loss of alpha motoneurons innervating the medial gastrocnemius muscle in a mouse model of amyotrophic lateral sclerosis. Exp. Neurol. 150:329-336.

18. Gregorevic, P., et al. 2004. Systemic delivery of genes to striated muscles using adeno-associated viral vectors. Nat. Med. 10:828-834.

19. Monahan, P.E., and Samulski, R.J. 2000. AAV vectors: is clinical success on the horizon? Gene Ther. 7:24-30.

20. Xiao, W., et al. 1999. Gene therapy vectors based on adeno-associated virus type 1.J. Virol. 73:3994-4003.

21. Hildinger, M., and Auricchio, A. 2004. Advances in AAV-mediated gene transfer for the treatment of inherited disorders. Eur. J. Hum. Genet. 12:263-271.

22. Wang, L.J., et al. 2002. Neuroprotective effects of glial cell line-derived neurotrophic factor mediated by an adeno-associated virus vector in a transgenic animal model of amyotrophic lateral sclerosis. J. Neurosci. 22:6920-6928.

23. Dobrowolny, G., et al. 2005. Muscle expression of a local Igf-1 isoform protects motor neurons in an ALS mouse model. J. Cell Biol. 168:193-199.

24. Delettre, C., et al. 2000. Nuclear gene OPA1, encoding a mitochondrial dynamin-related protein, is mutated in dominant optic atrophy. Nat. Genet.
26:207-210.

25. Alexander, C., et al. 2000. OPA1, encoding a dynamin-related GTPase, is mutated in autosomal dominant optic atrophy linked to chromosome 3q28. Nat. Genet. 26:211-215.

26. Zuchner, S., et al. 2004. Mutations in the mitochondrial GTPase mitofusin 2 cause CharcotMarie-Tooth neuropathy type 2A. Nat. Genet. 36:449-451.

27. Patel, H., et al. 2002. SPG20 is mutated in Troyer syndrome, an hereditary spastic paraplegia. Nat. Genet. 31:347-348.

28. Windpassinger, C., et al. 2004. Heterozygous missense mutations in BSCL2 are associated with distal hereditary motor neuropathy and Silver syndrome. Nat. Genet. 36:271-276.

29. Fink, J.K. 2002. Hereditary spastic paraplegia: the pace quickens. Ann. Neurol. 51:669-672.

30. Auricchio, A., Hildinger, M., O’Connor, E., Gao, G.P., and Wilson, J.M. 2001. Isolation of highly infectious and pure adeno-associated virus type 2 vectors with a single-step gravity-flow column. Hum. Gene Ther. 12:71-76.

31. Auricchio, A., et al. 2001. Exchange of surface proteins impacts on viral vector cellular specificity and transduction characteristics: the retina as a model. Hum. Mol. Genet. 10:3075-3081.

32. Gao, G.P., et al. 2002. Novel adeno-associated viruses from rhesus monkeys as vectors for human gene therapy. Proc. Natl. Acad. Sci. U. S. A. 99:11854-11859.

33. Gao, G., et al. 2000. Purification of recombinant adeno-associated virus vectors by column chromatography and its performance in vivo. Hum. Gene Ther. 11:2079-2091.

34. Previtali, S.C., et al. 2000. Epitope-tagged P(0) glycoprotein causes Charcot-Marie-Tooth-like neuropathy in transgenic mice. J. Cell Biol. 151:1035-1046.

35. Consiglio, A., et al. 2001. In vivo gene therapy of metachromatic leukodystrophy by lentiviral vectors: correction of neuropathology and protection against learning impairments in affected mice. Nat. Med. 7:310-316. 